Mário Sérgio ASSAYAG

JUNIOR';

Antonio Carlos PEDROSO';

Sebastião Gonçalves

FRANCO';

Suzane BODZIAK²;

Jaqueline de Cássia SILVA²

Correspondência para:

MARIO SÉRGIO ASSAYAG JUNIOR

Departamento de Patologia

Faculdade de Medicina Veterinária

Zootecnia-USP

Av. Prof. Orlando Marques de Paiva, 87

Cidade Universitária "Armando de Salles de

Oliveira"

05508-270 - São Paulo - SP

mario.assayag@intervet.com

Recebido para publicação: 27/08/2003 Aprovado para publicação: 15/03/2005

\title{
Efeito da duração do jejum pré-abate sobre peso corporal de frangos de corte aos $\mathbf{4 5}$ dias de idade
}

\author{
1 - Departamento de Patologia da Faculdade de Medicina Veterinária e \\ Zootecnia da USP, São Paulo - SP \\ 2 - Departamento de Zootecnia - UFPR, Curitiba-PR
}

\section{Resumo}

Foram utilizados 240 frangos de corte ROSS de ambos sexos criados separadamente, com objetivo de mensurar a perda de peso corporal em frangos de corte no pré-abate em 12 períodos de jejum alimentar com intervalo de 1 hora entre os períodos. As aves foram distribuídas em um delineamento inteiramente casualisado em esquema fatorial 2 x 12 x 10 (sexos x tratamentos x repetições). Foram escolhidas 10 aves de cada sexo para cada tratamento que se enquadrariam entre pesos mínimo e máximo, sendo para machos de $3.000 \pm 100$ gramas e para fêmeas de $2.400 \pm 100$ gramas. A análise da perda de peso corporal demonstrou que houve diferença significativa $(\mathrm{P}<0,05)$ entre os tratamentos, sendo que, ao aumentar o tempo de jejum, aumentou a perda de peso, sendo essa porcentagem maior para machos quando comparado às fêmeas.

\section{Introdução}

Uma das maneiras de diminuir a contaminação no abatedouro é submeter às aves a um período de jejum de alimento antes da apanha, carregamento e transporte. Durante o período de jejum, o trato digestivo é esvaziado e com isso haverá menor quantidade de material contaminante no abatedouro ${ }^{1}$. Além de que, segundo Mendes $^{2}$ ainda serve para melhorar a eficiência da produção ao evitar que alimento que não será transformado em carne seja fornecido às avess poucas horas antes do abate.

Durante muito tempo o jejum préabate vem sendo alvo de estudos e as dúvidas sempre foram em definir qual período de jejum que é suficiente para evitar riscos de contaminação no abatedouro e o menor comprometimento na perda de peso vivo e rendimento de carcaça.

Atualmente, outra grande preocupação dos pesquisadores é o bem estar dos animais e, conseqüentemente, melhorias para diminuir o impacto do estresse pré-abate que estão cada vez mais sendo o foco de estudos.

As pesquisas têm demonstrado que a utilização de tempo de jejum muito curto, os intestinos estariam repletos de ingesta, caso o tempo de jejum for excessivo, as aves tomarão muita água e ingerirão material de cama.

Vários pesquisadores estabeleceram como ideal o período de 8 a 12 horas de jejum alimentar, ${ }^{3,4,5}$.

Existe relação direta entre o tempo de jejum e a perda de peso das aves. As pesquisas têm indicado perdas de peso da ordem de 0,20 a $0,40 \%$ do peso vivo, por hora de jejum ${ }^{5}$.

À medida que aumenta a duração do período de jejum, aumenta a perda de peso das aves ${ }^{6,7,8}$. Segundo Denadai et al. ${ }^{9}$, isso é decorrência do menor conteúdo intestinal no momento do abate. Para Duke et al. ${ }^{10}$ além de confirmar a afirmação anterior, acrescenta que a perda de peso também é devida a desidratação ocorrida nos músculos e, segundo o pesquisador ${ }^{2}$, a desidratação da carcaça começa imediatamente após o inicio do jejum.

A maioria dos frigoríficos avícolas no 
Brasil utilizam o abate "just in time", metodologia que permite programar com maior precisão o tempo de jejum, as aves são submetidas ao jejum alimentar de 6 ou 8 horas, respectivamente para carregamentos durante o dia e a noite. Entretanto, a duração total de jejum normalmente fica entre 8 a 10 horas, em função da distância das granjas ao abatedouro.

Quanto à passagem do alimento pelo intestino, é relativamente rápida ${ }^{13}$. O tempo mínimo de passagem do alimento pelo intestino é de 2 a 2,5 horas Dansky e Hill ${ }^{12}$. Aproximadamente entre 50 a $75 \%$ da perda de peso vivo durante as primeiras 4 horas do jejum é devido à perda de água e matéria seca do conteúdo intestinal ${ }^{10}$.

A intensidade da perda de peso está relacionada com outros fatores como: tamanho das aves, condições de transporte e temperatura no ambiente de espera do abate ${ }^{6}$.

A época do ano deve ser levada em consideração, porque em dias muito quentes as aves não se alimentam de tarde quando o calor é mais intenso e se a suspensão da ração se der no final da tarde, o jejum vai ser mais prolongado do que o imaginado. O contrário também é questionado, em período de muito frio as aves se alimentam e tendem a ficar paradas, retardando a digestão e o risco de ocorrer papo cheio e contaminação da carcaça no abatedouro.

O objetivo desse trabalho foi mensurar a perda de peso corporal em frangos de corte em diferentes períodos de jejum alimentar no pré-abate.

\section{Materiais e Métodos}

O experimento foi realizado no Centro de Estações Experimentais do Cangüiri pertencente a UFPR. Foram testados 12 períodos de jejum alimentar no pré-abate (T1 a T12), respectivamente, correspondendo a um, dois, três, quatro, cinco, seis, sete, oito, nove, dez, onze e doze horas de jejum, colhendo a temperatura ambiental no momento da pesagem, que variou de 20 a $29^{\circ} \mathrm{C}$ durante todo jejum. Foram utilizados 240 aves de corte de 45 dias de idade, machos e fêmeas, da linhagem comercial AgROSS. As aves foram distribuídas por sexo em 48 boxes em delineamento inteiramente casualisado em esquema fatorial $2 \times 12 \times 10(\operatorname{sexos} \times$ tratamentos $\mathrm{x}$ repetições).

Foram escolhidas 10 aves de cada sexo para cada tratamento que se enquadraram entre peso mínimo e máximo, sendo para os machos de $3.000 \pm 100$ gramas e para as fêmeas de $2.400 \pm 100$. Atitude aplicada com o intuito do peso médio inicial ficar o mais homogêneo possível entre as aves do mesmo sexo para todos os tratamentos, já que a intensidade da perda de peso está relacionada com o tamanho das aves.

Todas as aves foram identificadas após a pesagem individual e a ração foi retirada para todos os tratamentos ao mesmo tempo, e após o período de jejum de cada tratamento, as aves foram pesadas novamente para mensurar a perda de peso corporal.

Os resultados foram submetidos ao programa estatístico ESTAT, pertencente ao Setor de Ciências Exatas da Universidade Estadual Paulista (UNESP), campus de Jaboticabal-SP e as médias pelo teste de Tukey a $5 \%$.

\section{Resutados e Discussões}

Os resultados da perda de peso corporal das aves em jejum alimentar no préabate estão apresentadas na tabela 1 e os resultados da \% de perda de peso corporal estão na tabela 2.

$\mathrm{Na}$ análise da perda de peso vivo e $\%$ dessa perda, houve diferença significativa $(\mathrm{P}<0,05)$ entre os tratamentos, sendo que, ao aumentar o tempo de jejum, aumentou a perda de peso. Resultados que estão concordantes com alguns autores $2,6,7,8,9$.

Afirmações que vão de encontro com 
Tabela 1 - Perda de peso corporal de machos e fêmeas aos 45 dias de idade em jejum alimentar no pré-abate. Curitiba, 2002.

\begin{tabular}{|c|c|c|c|}
\hline \multirow[t]{2}{*}{ Tratamento - Períodos de jejum (horas) } & \multicolumn{3}{|c|}{ Perda de peso corporal (g) } \\
\hline & Machos & Fêmeas & $\begin{array}{l}\text { Média dos dois } \\
\text { sexos }\end{array}$ \\
\hline $\mathrm{T} 12-12 \mathrm{~h}$. & $179 a^{1}$ & $134 \mathrm{a}$ & $157 \mathrm{a}$ \\
\hline T11 - $11 \mathrm{~h}$. & $173 \mathrm{a}$ & $126 \mathrm{a}$ & $149 \mathrm{ab}$ \\
\hline $\mathrm{T} 10-10 \mathrm{~h}$. & $155 \mathrm{~b}$ & $120 \mathrm{ab}$ & $135 \mathrm{~b}$ \\
\hline T9 $-9 \mathrm{~h}$ & $124 \mathrm{bc}$ & $106 \mathrm{bc}$ & $115 c$ \\
\hline $\mathrm{T} 8-8 \mathrm{~h}$ & $104 \mathrm{~cd}$ & $88 \mathrm{~cd}$ & $96 \mathrm{~d}$ \\
\hline $\mathrm{T} 7-7 \mathrm{~h}$. & 86 de & $83 \mathrm{~cd}$ & 84 de \\
\hline T6 - $6 \mathrm{~h}$. & 85 de & 73 de & 79 de \\
\hline $\mathrm{T} 5-5 \mathrm{~h}$ & 74 ef & 64 def & $69 \mathrm{ef}$ \\
\hline $\mathrm{T} 4-4 \mathrm{~h}$. & $68 \mathrm{ef}$ & 47 efg & $57 \mathrm{fg}$ \\
\hline $\mathrm{T} 3-3 \mathrm{~h}$ & $52 \mathrm{fg}$ & $40 \mathrm{fg}$ & $46 \mathrm{gh}$ \\
\hline $\mathrm{T} 2-2 \mathrm{~h}$ & $37 \mathrm{~g}$ & $30 \mathrm{~g}$ & $33 \mathrm{~h}$ \\
\hline $\mathrm{T} 1-1 \mathrm{~h}$. & $33 \mathrm{~g}$ & $28 \mathrm{~g}$ & $31 \mathrm{~h}$ \\
\hline
\end{tabular}

1- Médias seguidas de letras diferentes na mesma coluna diferem pelo teste de Tukey $(P<0,05)$

Lyon, Papa e Wilson Jr. ${ }^{1}$ que demonstraram haver relação linear significativa da perda de peso com o tempo de jejum. O mesmos autores, avaliaram o efeito de períodos de jejum de $0 ; 8 ; 16$ e 24 horas sobre a perda de peso e observaram que a perda de peso aumentou linearmente com a duração do jejum, respectivamente de $0 ; 2,94 ; 4,32$ e $5,61 \%$. Nesta pesquisa obteve-se em 8 horas de jejum perda de peso de 3,48\% para os machos e 3,65\% para as fêmeas e 3,55\% de média para os dois sexos, em temperatura ambiente que variou de 24 a $29^{\circ} \mathrm{C}$.

Sarica, Karacay e $\mathrm{Cam}^{13}$ submeteram frangos de corte com 50 dias de idade a períodos de jejum de $0 ; 2 ; 4 ; 6 ; 8$ e 10 horas e encontraram, respectivamente, as seguintes perdas $0 ; 0,88 ; 1,86 ; 2,14 ; 2,82$ e $3,56 \%$. Verificou-se neste trabalho com os mesmos períodos, com exceção do 0 , média dos dois sexos, respectivamente, as perdas de 1,41 ; 2,$09 ; 2,88 ; 3,55$ e $4,99 \%$.

Rosa et al. ${ }^{14}$, medindo o efeito da temperatura e duração do jejum pré-abate sobre a perda de peso corporal em frangos de corte, obtiveram para os períodos de jejum de 3; 6; 9 e 12 horas, respectivamente, as médias com os respectivos desvios padrão de $40 \pm$ 3,8g; $62 \pm$ 3,7g; $104 \pm$ 3,7g; $151 \pm$ 3,9 g. Este trabalho com os mesmos períodos de jejum conseguiu para os dois sexos, respectivamente, as perdas corporais de 46g; $79 \mathrm{~g} ; 115 \mathrm{~g}$ e $157 \mathrm{~g}$, em temperatura ambiente que variou de 20 a $29^{\circ} \mathrm{C}$.

Provavelmente, a grande parte da perda de peso corporal nas aves, acontece nas primeiras 4 horas, isto ocorre devido ao esvaziamento do conteúdo intestinal ${ }^{10}$.

\section{Conclusões}

Conclui-se que, nas condições deste experimento, à medida que aumenta a duração do período de jejum, aumenta a \% de perda de peso corporal das aves, sendo essa porcentagem maior para os machos do que para as fêmeas.

A duração do jejum alimentar no préabate precisa ser considerada na determinação do período adequado de jejum, para se conseguir a menor porcentagem de perda de peso corporal, 
Tabela 2 - Porcentagem de perda de peso corporal de machos e fêmeas aos 45 dias de idade, em jejum alimentar no pré-abate. Curitiba, 2002

\begin{tabular}{|c|c|c|c|}
\hline \multirow[t]{2}{*}{ Tratamento- Períodos de jejum (horas) } & \multicolumn{3}{|c|}{$\%$ Perda de peso corporal } \\
\hline & Machos & Fêmeas & $\begin{array}{l}\text { Média dos dois } \\
\text { sexos } \\
\end{array}$ \\
\hline T12-12 h. & $5,97 \mathrm{a}^{1}$ & $5,55 \mathrm{a}$ & $5,80 \mathrm{a}$ \\
\hline T11 - $11 \mathrm{~h}$. & $5,81 \mathrm{a}$ & 5,19 a & $5,51 \mathrm{a}$ \\
\hline $\mathrm{T} 10-10 \mathrm{~h}$. & $571 \mathrm{a}$ & $4,97 \mathrm{a}$ & $4,99 \mathrm{ab}$ \\
\hline T9 - $9 \mathrm{~h}$ & $4,11 \mathrm{~b}$ & $4,39 \mathrm{ab}$ & $4,42 \mathrm{bc}$ \\
\hline T8 $-8 \mathrm{~h}$. & $3,48 \mathrm{bc}$ & $3,65 \mathrm{~b}$ & $3,55 \mathrm{bc}$ \\
\hline $\mathrm{T} 7-7 \mathrm{~h}$ & $2,83 \mathrm{~cd}$ & $3,38 \mathrm{bc}$ & $3,07 \mathrm{~cd}$ \\
\hline $\mathrm{T} 6-6 \mathrm{~h}$ & $238 \mathrm{~cd}$ & $2,98 \mathrm{~cd}$ & $2,88 \mathrm{de}$ \\
\hline $\mathrm{T} 5-5 \mathrm{~h}$ & 2,47 cde & $2,58 \mathrm{~cd}$ & 2,52 de \\
\hline $\mathrm{T} 4-4 \mathrm{~h}$. & 2,27 de & 1,92 cde & $2,09 \mathrm{e}$ \\
\hline $\mathrm{T} 3-3 \mathrm{~h}$ & 1,73 ef & 1,63 de & $1,68 \mathrm{ef}$ \\
\hline $\mathrm{T} 2-2 \mathrm{~h}$. & 1,22 ef & 1,21 ef & $1,41 \mathrm{f}$ \\
\hline T1 - 1 h. & $1,10 \mathrm{f}$ & $1,12 \mathrm{f}$ & $1,13 \mathrm{f}$ \\
\hline
\end{tabular}

${ }^{1}$ Médias seguidas de letras diferentes na mesma coluna diferem pelo teste de Tukey $(\mathrm{P}<0,05)$

nenhum transtorno no abatedouro, caminhões transportadores que ficam principalmente, naqueles que não praticam estacionados muito tempo na área de espera o "just in time" e que trabalham com antes de descarregar no abatedouro.

\section{Effect of the duration of pre-slaughter feed withdrawal on body weight of broilers chickens at $\mathbf{4 5}$ days of age}

\section{Abstract}

Were used 240 broilers Ross of both sexes created separately, with objective to measure the loss of corporal weight in broilers in the preslaughter in 12 periods of withdrawal with interval of 1 hour between the periods. The birds were distributed in experimental desing completely randomized with a $2 \times 12 \times 10$ factorial (sexes x treatments $\mathrm{x}$ repetitions). We chose 10 birds of each sex for each treatment between a minimum weight and a maximum weight. For the males it was certain $3.000 \pm 100$ grams and for the females $2.400 \pm 100$ grams. The analysis of the loss of corporal weight demonstrated that there was significant difference $(\mathrm{P}<0,05)$ among the treatments, and, when increasing the time of withdrawal, it also increased the weight loss, being that larger percentage for the males when compared with the females.

\section{Referências}

1 LYON, C. E.; PAPA, C. M.; WILSON Jr., R. L. Effect of feed withdrawal on yields, muscle $\mathrm{pH}$, and texture of

\section{Key-words:}

Pre-Slaughter feed withdrawal. Broilers chickens. 
Revista Brasileira de Ciência Avícola, v. 3, p. 199-209, 2001.

3 BARTOV, I. Effect of feed withdrawal on yield, fat content, and fatty acid composition of various tissues in broilers. In: WORLD'S POULTRY CONGRESS, 19.,1992, Proceedings... v. 3, p. 195-199.

4 VEERKAMP, C. H. Future research for pre-slaughter handling, stunning and related processes. In: WORLD'S POULTRY CONGRESS, 19., 1992. Proceedings: v. 2, p. 352-359.

5 ROSA, P. S.; ÁVILA, V. S.; JAENISCH, F. R. F. Restrição alimentar em frangos de corte: como explorar suas potencialidades. Concórdia: Suínos e Aves, 2000. 4 p. (Embrapa Suínos e Aves. Comunicado Técnico, 250).

6 CHEN, T. C. et al. The effect of extended holding time, temperature, and dietary energy on yields of broilers. Poultry Science, v. 62, p. 1566-1571, 1983.

7 RASMUSSEN, A. L.; MAST, M. G. Effect of feed withdrawal on composition and quality of meat. Poultry Science, v. 68, p. 1109-1113, 1989.

8 WABECK, C. J. Feed and water withdrawal time relationship to processing yield and potential fecal contamination of broilers. Poultry Science, v. 51, p. 1119-1121, 1972.

9 DENADAI, J. C. et al. Efeito do tempo de jejum préabate sobre o rendimento de carcaça e qualidade da carne de peito de frangos de corte. In: REUNIÃO ANUAL DA SOCIEDADE BRASILEIRA DE ZOOTECNIA, 38., 2001, Piracicaba. Anais...Piracicaba, 2001. p. 394-395.

10 DUKE, G. E.; MAUREEN, B.; NOLL, S. Optimum duration of feed and water removal prior to processing in order to reduce the potential for fecal contamination in turkeys. Poultry Science, v. 76, n. 3, p. 516-522, 1997.

11 HILLERMAN, J. P.; KRATZER, F. H.; WILSON, W. D. Food passage through chickens and turkeys and same regulating factors. Poultry Science, v. 32, p. 332 337, 1953.

12 DANSKY, L. M.; HILL, F. W. Application of the chromic oxide indicator method to balance studies of growing chickens. Journal of Nutrition, v. 47, p. 449453, 1952.

13 SARICA, M.; KARACAY, N.; CAM, N. A. Effects of various feed withdrawal periods on yields and some cutting and carcass traits of broilers. Turk Veterinerlik ve Haivancelik Dergisi, v. 19, n. 4, p. 303-307, 1995.

14 ROSA, P. S. et al. Efeito da temperatura e duração de jejum pré-abate sobre indicadores de estresse em frangos de corte abatidos aos 35 e 49 dias de idade. Revista Brasileira de Ciência Avícola, p. 9, Campinas, 2002. Suplemento 4. 